\title{
Oncogenic rearrangements driving ionizing radiation-associated human cancer
}

\author{
Massimo Santoro and Francesca Carlomagno
}

Dipartimento di Medicina Molecolare e Biotecnologie Mediche, Università di Napoli "Federico II," Naples, Italy.

\begin{abstract}
The Chernobyl nuclear disaster has caused a remarkable increase in radiation-induced papillary thyroid carcinoma in children and young adults. In this issue of the JCI, Ricarte-Filho and colleagues demonstrate that chromosomal rearrangements are the oncogenic "drivers" in most postChernobyl carcinomas and that they often lead to unscheduled activation of the MAPK signaling pathway. These findings represent a major step forward in our understanding of radiation-induced carcinogenesis and suggest various hypotheses about the mechanisms underlying the formation and selection of gene rearrangements during cancer cell evolution.
\end{abstract}

\section{Repercussions of the Chernobyl disaster}

Papillary thyroid cancer (PTC) is the most common malignancy of the thyroid gland and can be associated with ionizing radiation $(1,2)$. The accident at the Chernobyl nuclear power plant on April 26, 1986, in Ukraine contaminated the regions surrounding the reactor with large amounts of iodine 131 and other short-lived iodine isotopes (2). As early as 4 years after the disaster, there was a sharp rise in PTC incidence, with more than 4,000 cases diagnosed in Belarus, Russia, and Ukraine, among patients who were children or adolescents at the time of the accident (2). Given the rarity of thyroid cancer in young people, it is feasible that a large fraction of post-Chernobyl PTCs were the result of radiation exposure (2).

\section{Oncogenic drivers of PTC}

In approximately $70 \%$ of "sporadic" (e.g., without a reported association with radiation exposure) PTC cases, cancer-driving genetic lesions have been identified (Figure 1). PTC development frequently $(\sim 50 \%$ of cases) involves point mutations in the BRAF serine/threonine kinase; however, sometimes gene rearrangements of the receptor tyrosine kinase $R E T$ (with rearrangements referred to herein as $R E T$ / $P T C)$ and the neurotrophic tyrosine kinase receptor type 1 (NTRK1) are identified. These rearrangements result in the in-

Conflict of interest: The authors have declared that no conflict of interest exists.

Citation for this article: $J$ Clin Invest.

2013;123(11):4566-4568. doi:10.1172/JCI72725. frame fusion of the RET or NTRK1 kinase domain to the $\mathrm{N}$ terminus of heterologous proteins, a process that leads to unscheduled activation of the enzyme $(1,3)$. Similar to follicular thyroid carcinoma, the follicular variant of PTC displays activating mutations of small GTPase RAS genes or, occasionally, gene rearrangements that target $P P A R G$, a member of the nuclear hormone receptor family (1).

Unlike sporadic cases, post-Chernobyl carcinomas display a high prevalence of gene rearrangements, mainly nuclear receptor coactivator 4-RET (NCOA4-RET) (RET/PTC3) fusion, and a low prevalence of BRAF point mutations (4-6). Together with experimental data showing that exposure to $\mathrm{X}$-rays induces RET/PTC fusions in thyroid cells (7), these findings suggest the existence of a mechanistic link between ionizing radiation exposure and PTC. Namely, radioiodine exposure promotes oncogeneactivating gene rearrangement, which results in PTC formation. Conflicting with this view of radiation-induced PTC, previous studies have demonstrated that childhood PTCs without a reported history of radiation exposure also feature a high prevalence of $R E T$ rearrangements and a low prevalence of BRAF point mutations (8-11). These findings challenge the notion that radiation exposure increases the frequency of oncogenic rearrangements and suggest instead that it is patient age that promotes the high frequency of rearrangements.

\section{Radiation exposure and PTC- associated fusion oncogenes}

In this issue of the JCI, using RT-PCR, mass spectrometry, and next-generation
RNA-sequencing, Ricarte-Filho and colleagues demonstrate that the majority of PTCs (22 out of 26 tumors, $84 \%$ ) that occurred in patients who were less than 10 years old at the time of the Chernobyl accident and lived in contaminated areas harbored fusion oncogenes. They found fifteen cases with RET/PTC, 1 case with translocated protein region-NTRK1 (TPR-NTRK1), two cases with ets variant 6-NTRK3 (ETV6-NTRK3), one case with A kinase anchor protein 9-BRAF (AKAP9-BRAF), one case with acylglycerol kinase-BRAF (AGK-BRAF), one case with cAMP-responsive element binding protein 3-like 2-PPARG (CREB3L2-PPARG), and one case with paired box 8-PPARG (PAX8-PPARG) (Figure 1 and ref. 12). Interestingly, these fusions often resulted from intrachromosomal rearrangements targeting chromosome 10 (RET/PTC1, RET/PTC3, RET/PTCA-3), chromosome 1 (TPR-NTRK1), or chromosome 7 (AKAP9BRAF, AGK-BRAF). Only a minority of post-Chernobyl PTCs featured point mutations or indels in BRAF or thyroidstimulating hormone receptor (TSHR) (12). Conversely, thyroid cancers from patients of the same age without a history of radiation exposure displayed a lower prevalence of fusion oncogenes and a higher prevalence of point mutations compared with radiation-associated carcinomas (12). It should be noted that the collection of oncogenic lesions in sporadic childhood thyroid cancers was not complete, since the oncogenic driver lesions were identified in only 18 out of 27 of the cases in this study (12).

Overall, the study by Ricarte-Filho and colleagues demonstrates that radiation exposure caused a selective increase of oncogenic driver events generated by gene rearrangements compared with point mutations (12). This work has led to the discovery of two previously undescribed oncogenic fusion events that are involved in thyroid cancer formation, namely, ETV6-NTRK3 and AGK-BRAF fusion events. ETV6-NTRK3 fusion events were previously 


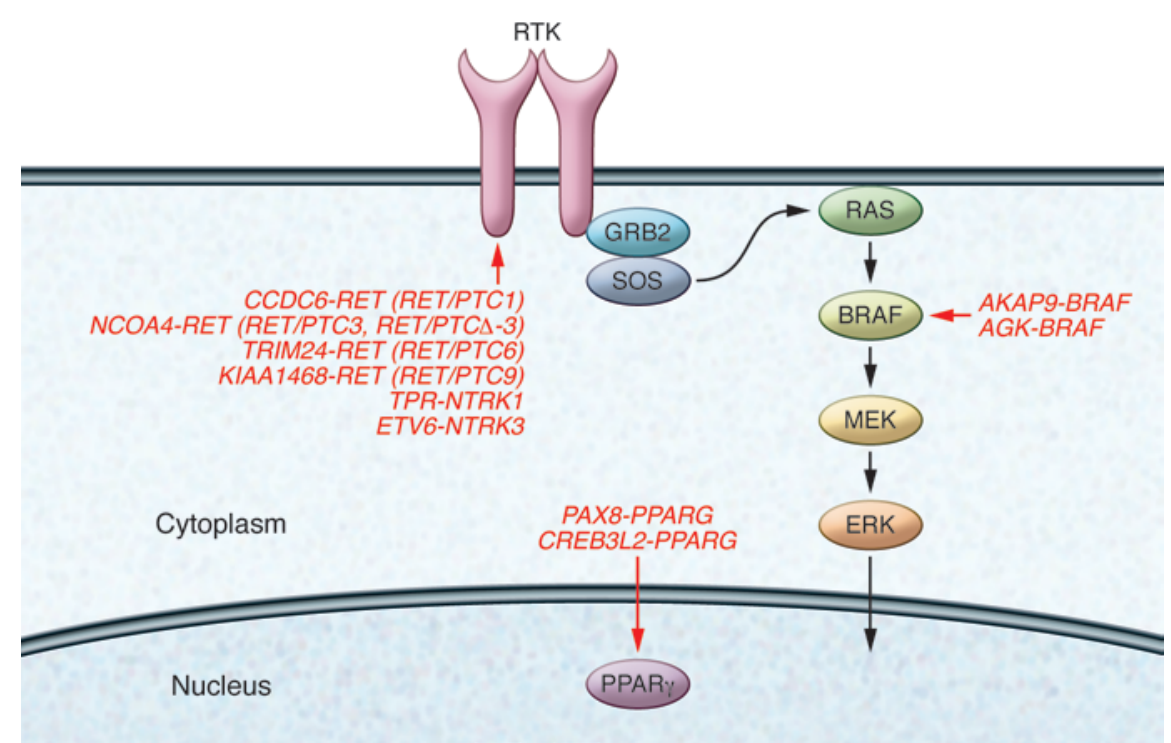

Figure 1

A summary of gene rearrangements identified in post-Chernobyl PTCs in the RicarteFilho study (red) (12). Most of them target components of the MAPK signaling cascade and include rearrangements of RET (RET/PTC) or NTRK1 and NTRK3 receptor tyrosine kinases (RTKs), or BRAF kinase. An exception is represented by rearrangements affecting the PPARG steroid hormone receptor.

identified, albeit with different breakpoints, in various nonthyroid tumors (13), further confirming the oncogenic potential of constitutive NTRK3 activation. The $A G K-B R A F$ fusion event identified in this study appears to be a unique recombination, although $B R A F$ rearrangement with partners other than $A G K$ is an established mechanism of $B R A F$ oncogenic conversion in other human cancers (14).

\section{Radiation promotes proximity- dependent oncogenic fusion events} The study by Ricarte-Filho et al. also demonstrated that, although post-Chernobyl PTCs had a compelling prevalence of oncogenic gene fusion events, the total number of gene rearrangements, as detected by low-pass whole-genome sequencing, did not differ between radiation-exposed and sporadic cases (12). These data suggest an intriguing concept: it is the nature of the rearrangements rather than their overall abundance that differentiates sporadic from radiation-induced thyroid cancers; therefore, only those rearrangements that generate cancer-driving fusion oncoproteins are selectively increased in radiation-exposed patients. The fact that the postradiation neoplastic clones did not display an overall excess of random chromosomal aberrations indicates that only a discrete set of gene rearrangements was induced by radiation in the initiated cells. Radiation can result in double-stranded DNA breaks that when not correctly repaired can give rise to gene fusion events. To generate a gene rearrangement, partner genes must be brought into close spatial proximity; therefore, genes that are on the same chromosome have the highest chance of fusing together. Nikiforov and colleagues provided strong evidence that this mechanism governs RET and BRAF gene rearrangements in thyroid cancer by demonstrating proximity, specifically in thyroid cells, between RET and coiled-coil domain containing 6 (CCDC6) (RET/PTC1) (15), RET and NCOA4 (RET/PTC3) (16), and $A K A P 9$ and BRAF (17). Similarly, NTRK1 and TPR loci often overlap in interphase chromatin (18). It would be interesting to learn whether these features are applicable to the other radiation-associated gene rearrangements identified by Ricarte-Filho and coworkers. Moreover, since post-Chernobyl carcinomas occurred in young patients and in geographic areas with reduced iodine supply $(19,20)$, it would also be interesting to determine whether age and iodine concentration influence thyrocyte chromatin organization. In this context, it is plausible that, due to gene proximity, radiation exposure promotes the formation of thy- roid cancer-driving gene fusion events. On the other hand, it cannot be excluded that radiations are also able to induce irrelevant ("passenger") or even detrimental gene rearrangements in individual thyrocytes. However, these lesions, being unable to confer any selective advantage, would not be detectable in the tumor bulk.

\section{Radiation-associated oncogenes commonly trigger the MAPK pathway} It is compelling that all but two of the oncogenic rearrangements identified by Ricarte-Filho and colleagues lead to constitutive activation of the MAPK signaling cascade (Figure 1 and ref. 12). This lends further support to the notion that this particular pathway plays a major role in thyroid carcinogenesis $(1,3)$. An important exception to this paradigm is represented by the two samples that featured PPARG rearrangements (CREB3L2-PPARG, $P A X 8-P P A R G)$, confirming the previous observations of Leeman-Neill and colleagues (20). These samples were from follicular rather than classic-variant PTCs, which supports previous studies that correlate PPARG genetic lesions with this particular morphological PTC subtype (1). Moreover, the follicular-derived PTC samples featured a PPAR $\gamma$-driven rather than a MAPK-driven transcriptional signature. These findings suggest that PPARG rearrangements, though most commonly identified in cancers unrelated to radiation (1), may also be triggered by exposure to ionizing radiations.

\section{Future implications}

In conclusion, the study by Ricarte-Filho et al. enables us to draw the genetic portrait of a human cancer caused by a well-defined etiological factor, thereby paving the way for studies aimed at understanding how ionizing radiation induces chromosomal rearrangements in cancer cells.

\section{Acknowledgments}

This study was supported in part by grants AIRC 10575 and AIRC 11822 from the Italian Association for Cancer Research. We apologize to the many authors whose work has not been cited due to space limitation.

Address correspondence to: Massimo Santoro, Dipartimento di Medicina Molecolare e Biotecnologie Mediche, Università di Napoli “Federico II," via S Pansini 5, 80131 Naples, Italy. Phone: 39.081.7463056; Fax: 39.081.7463037; E-mail: masantor@unina.it. 
1. Nikiforov YE, Nikiforova MN. Molecular genetics and diagnosis of thyroid cancer. Nat Rev Endocrinol. 2011;7(10):569-580.

2. Williams D. Radiation carcinogenesis: lessons from Chernobyl. Oncogene. 2008;27(suppl 2):S9-S18.

3. Santoro M, Carlomagno F. Drug insight: Smallmolecule inhibitors of protein kinases in the treatment of thyroid cancer. Nat Clin Pract Endocrinol Metab. 2006;2(1):42-52.

4. Rabes HM, Klugbauer S. Molecular genetics of childhood papillary thyroid carcinomas after irradiation: high prevalence of RET rearrangement Recent Results Cancer Res. 1998;154:248-264.

5. Nikiforova MN, et al. Low prevalence of BRAF mutations in radiation-induced thyroid tumors in contrast to sporadic papillary carcinomas. Cancer Lett. 2004;209(1):1-6.

6. Lima J, et al. BRAF mutations are not a major event in post-Chernobyl childhood thyroid carcinomas. J Clin Endocrinol Metab. 2004;89(9):4267-4271.

7. Ito $T$, et al. In vitro irradiation is able to cause RET oncogene rearrangement. Cancer Res. 1993; 53(13):2940-2943

8. Nikiforov YE, Rowland JM, Bove KE, MonforteMunoz H, Fagin JA. Distinct pattern of ret oncogene rearrangements in morphological variants of radia- tion-induced and sporadic thyroid papillary carcinomas in children. Cancer Res. 1997;57(9):1690-1694.

9. Bongarzone I, et al. Age-related activation of the tyrosine kinase receptor protooncogenes RET and NTRK1 in papillary thyroid carcinoma. J Clin Endocrinol Metab. 1996;81(5):2006-2009.

10. Fenton CL, Lukes Y, Nicholson D, Dinauer CA Francis GL, Tuttle RM. The ret/PTC mutations are common in sporadic papillary thyroid carcinoma of children and young adults. J Clin Endocrinol Metab. 2000;85(3):1170-1175.

11. Kumagai A, et al. Low frequency of BRAFT1796A mutations in childhood thyroid carcinomas. J Clin Endocrinol Metab. 2004;89(9):4280-4284.

12. Ricarte-Filho JC, et al. Identification of kinase fusion oncogenes in post-Chernobyl radiation-induced thyroid cancers. J Clin Invest. 2013 123(11):4935-4944.

13. Cetinbas N, et al. Mutation of the salt bridge-forming residues in the ETV6 SAM domain interface blocks ETV6-NTRK3 induced cellular transformation [published online ahead of print June 24, 2013]. J Biol Chem. doi:10.1074/jbc.M113.475301.

14. Ciampi R, et al. Oncogenic AKAP9-BRAF fusion is a novel mechanism of MAPK pathway activation in thyroid cancer. J Clin Invest. 2005;115(1):94-101.
15. Nikiforova MN, Stringer JR, Blough R, Medvedovic M, Fagin JA, Nikiforov YE. Proximity of chromosomal loci that participate in radiationinduced rearrangements in human cells. Science. 2000;290(5489):138-141.

16. Gandhi M, Medvedovic M, Stringer JR, Nikiforov YE. Interphase chromosome folding determines spatial proximity of genes participating in carcinogenic RET/PTC rearrangements. Oncogene. 2006;25(16):2360-2366.

17. Gandhi M, Evdokimova V, Nikiforov YE. Frequency of close positioning of chromosomal loci detected by FRET correlates with their participation in carcinogenic rearrangements in human cells. Genes Chromosomes Cancer. 2012;51(11):1037-1044.

18. Roccato E, et al. Proximity of TPR and NTRK1 rearranging loci in human thyrocytes. Cancer Res. 2005;65(7):2572-2576.

19. Cardis E, et al. Risk of thyroid cancer after exposure to $131 \mathrm{I}$ in childhood. J Natl Cancer Inst. 2005; 97(10):724-32.

20. Leeman-Neill RJ, et al. RET/PTC and PAX8/PPAR $\gamma$ chromosomal rearrangements in post-Chernobyl thyroid cancer and their association with iodine-131 radiation dose and other characteristics. Cancer. 2013;119(10):1792-1799.

\title{
"N of 1" case reports in the era of whole-genome sequencing
}

\author{
A. Rose Brannon ${ }^{1,2}$ and Charles L. Sawyers ${ }^{2,3}$ \\ 1Department of Pathology, ${ }^{2}$ Human Oncology and Pathogenesis Program, and ${ }^{3}$ Howard Hughes Medical Institute, \\ Memorial Sloan-Kettering Cancer Center, New York, New York, USA.
}

\begin{abstract}
Prostate cancer has a range of clinical outcomes, from complete remission in response to treatment to death as a result of aggressive metastasis. Prognosis for individuals with prostate cancer is not readily predictable, and new diagnostics will be useful for treatment strategy determination. In this issue of the JCI, Haffner and colleagues use comprehensive tumor genome sequencing to investigate the origin of genetic mutations underlying a case of lethal prostate cancer. Surprisingly, the lethal clone in this individual arose from a tumor focus that is typically considered very low risk based on histology. Their report highlights the need to collect and curate " $N$ of 1 " cases to develop a database that can be used for clinical decision making.
\end{abstract}

\section{The case of the lethal clone}

Scientists are detectives at heart. When Haffner and colleagues learned about a case of lethal metastatic prostate cancer with evidence dating back 17 years, they had to take the case (1). Using an approach similar to one previously employed to follow pancreatic cancer progression (2), the authors began with comprehensive

Conflict of interest: Charles L. Sawyers serves on the Board of Directors of Novartis Pharmaceuticals and on the Scientific Advisory Boards of Agios, Beigene, Blueprint, Housey Pharmaceuticals, Nextech, and Tracon.

Citation for this article: J Clin Invest. 2013;

123(11):4568-4570. doi:10.1172/JCI70935. genome sequencing of metastatic tumor deposits recovered at autopsy. This analysis yielded evidence of mutations in several well-documented prostate cancer genes, such as tumor suppressor PTEN, tumor protein p53 (TP53), speckle-type POZ protein $(S P O P)$, ATP-dependent helicase $(A T R X)$, and androgen receptor $(A R)(1)$, all of which are known to be recurrently altered in end-stage, castration-resistant prostate cancer. Because this patient's primary tumor had been surgically excised (and saved) 17 years earlier, the authors had a unique opportunity to ask the "whodunit" question. Which, if any, of these mutations were present in the primary tumor? How did this constellation of mutations evolve over time as the patient suffered multiple relapses (with accompanying tissue biopsies) during 17 years of treatment with various interventions? Answering these questions offered the potential for new insights into prostate cancer progression, drug resistance mechanisms, and perhaps opportunities to develop molecular diagnostics.

Histological examination of the primary tumor revealed multiple regions of highgrade (Gleason 4) tumor, a small focus of lower-grade (Gleason 3) disease, and a single lymph node metastasis (1). It is well established that patients with highvolume, high-grade primary disease have an increased risk of recurrence; therefore, this patient's subsequent clinical course of metastatic prostate cancer is not surprising. It was surprising that the lethal clone, defined by the presence of the same PTEN, TP53, and SPOP mutations recovered at autopsy, originated from the small, lowgrade Gleason 3 focus, and not from the much more substantial, high-volume Gleason 4 tumors, which did not harbor PTEN, 